\title{
DETERMINACIÓN ESTRUCTURAL DE COMPUESTOS FENÓLICOS DEL EXTRACTO HIDROALCOHÓLICO DE
}

\author{
Geranium ruizii Hieron
}

\author{
STRUCTURAL DETERMINATION OF PHENOLIC COMPOUNDS \\ HYDROALCOHOLIC EXTRACT Geranium ruizzi Hieron
}

\author{
${ }^{1}$ Gustavo Adolfo Fernández Rebaza; 'Maria Eugenia Quiñones Huayaney; 'Jorge Luis Tolentino Chávez; \\ 'Yoselin Milagros Chambi Velasquez; ' José Miguel Ayme Huamaní; ' Pablo Enrique Bonilla Rivera
}

\begin{abstract}
RESUMEN
La diabetes mellitus se considera como una de las enfermedades crónicas más complicadas de tratar. La Organización Mundial de la Salud (OMS) ha estimado que para el 2030 el número de diabéticos será de 32,9 millones de personas en el mundo. Las plantas medicinales con capacidad antidiabética son una fuente de nuevos compuestos en el tratamiento de la diabetes. Geranium lechleri Knuth "pasuchaca", es una de ellas. De todos los compuestos fenólicos, el grupo de los flavonoides es el más extendido en la naturaleza y son los que poseen una mayor actividad antioxidante. Varios estudios han verificado el efecto hipoglucemiante de la "pasuchaca" como inhibidor de la $\alpha$-glucosidasa, la proteína que degrada el glucógeno en la síntesis de la glucosa. Se utiliza desde hace muchos años por el ser humano sin que se produzcan efectos colaterales, se supone que su ingesta no implica riesgo para la salud. El propósito de la presente investigación fue la detección, aislamiento y determinación estructural de los principales compuestos fenólicos del extracto hidroalcohólico de Geranium ruizii Hieron "pasuchaca"; para lo cual se elaboró el extracto hidroalcohólico de la planta completa de "pasuchaca", se realizó su estudio fitoquímico y mediante técnicas cromatográficas, se aislaron y purificaron compuestos fenólicos, luego se propuso mediante espectroscopía ultravioleta visible la estructura de algunos flavonoides encontrados.
\end{abstract}

Palabras clave: Geranium ruizii, pasuchaca, diabetes, compuestos fenólicos, flavonoides, espectroscopia UV/vis.

\begin{abstract}
Diabetes mellitus is considered as one of the most difficult to treat chronic diseases. The World Health Organization (OMS) has estimated that by 2030 the number of diabetics will be 32.9 million people worldwide. Medicinal plants with antidiabetic capabilities are a source of new compounds in the treatment of diabetes. Geranium lechleri Knuth "pasuchaca" is one of them. Of all the phenolic compounds, the group of flavonoids is the most widespread in nature and are those with higher antioxidant activity. The hypoglycemic effect according to several studies on this plant, it has been verified that the "pasuchaca" plant species is attributed as an inhibitor of $\alpha$ - glucosidase protein that degrades glycogen synthesis from glucose. Used for many years by humans without side effects occur, it is assumed that their intake no risk to health. The aim of this study was the detection, isolation and structural determination of major phenolic compounds in hydroalcoholic extract of Geranium ruizii Hieron "pasuchaca"; for which the hydroalcoholic extract of the whole plant of "pasuchaca" was developed, its phytochemical study was carried out by chromatographic techniques and isolated and purified phenolic compounds, then UV visible spectroscopy proposed by the structure of some flavonoids found.
\end{abstract}

Keywords: Geranium ruizii, pasuchaca, diabetes, phenolics, flavonoids, UV/vis spectroscopy.

\section{INTRODUCCIÓN}

La diabetes es una enfermedad metabólica crónica que aparece debido a que el páncreas no fabrica la cantidad de insulina que el cuerpo humano necesita. La insulina, una hormona producida por el páncreas, es la principal sustancia responsable del mantenimiento de los valores adecuados de azúcar en la sangre.

La diabetes mellitus se considera como una de las enfermedades crónicas más complicadas de tratar. La Organización Mundial de la Salud (OMS) ha estimado que para el 2030 el número de diabéticos será de 32,9 millones de personas en el mundo. El Perú representa el 0,11\% del mercado farmacéutico mundial. Hay que tener en cuenta que más del $50 \%$ de los peruanos se ubican en el nivel de pobreza y más del $20 \%$ en extrema pobreza, sin posibilidades de acceso a medicamentos convencionales. Por lo tanto, se hace indispensable la búsqueda de medicamentos eficaces y de bajo costo. ${ }^{2}$

De todos los compuestos fenólicos, el grupo de los flavonoides es el más extendido en la naturaleza y dentro de ellos, los flavonoles son los que poseen una mayor actividad antioxidante. Por un lado, disminuyen las tasas de colesterol y de LDL oxidada debido a sus propiedades antioxidantes, 
Fernández G. et al., Determinación Estructural de Compuestos Fenólicos del Extracto Hidroalcohólico de Geranium ruizii Hieron

como fuertes quelantes de metales y como donadores de hidrógeno (a través de los grupos hidroxilo). Así, el grado de actividad antioxidante se correlaciona con el número de grupos hidroxilo. Por ello, los ortodifenoles son buenos antioxidantes, mientras que compuestos monofenol, como el tirosol, no lo son tanto. ${ }^{2}$

Las plantas medicinales con capacidad antidiabética son una fuente de nuevos compuestos en el tratamiento de la diabetes. Geranium lechleri Knuth"pasuchaca"es una de ellas, oriunda del Perú utilizada desde hace mucho tiempo para controlar los niveles de glucosa. Esta planta se cultiva en la sierra del Perú, a más de 3500 metros sobre el nivel del mar, se puede encontrar en los departamentos de Cajamarca, La Libertad, Arequipa, Cerro de Pasco y Ancash.

Según un estudio de cuti cuti existen algunos principios activos aislados de las plantas como: Los flavonoides y alcaloides que se encuentran en gran proporción en esta y otras plantas con efecto hipoglucemiante, entre los cuales tenemos a los flavonoides: rutina y quercetina que cuentan con investigaciones probadas de su efecto hipoglucemiante; la quercetina ejerce un efecto hipoglucemiante, inhibiendo a la enzima aldosa reductasa, la cual está relacionada con la producción de polioles, responsables de los efectos crónicos en la diabetes $y$, por sus propiedades antioxidantes, reduce la incidencia de enfermedades cardiovasculares, incluyendo las trombosis vasculares. $^{4,5}$

El Geranium ruižii Hieron en su composición química presenta glucósidos (cianogenéticos antraquinónicos, anticiánicos, saponínicos) gomas, mucílagos, taninos, saponinas, clorofila, grasas, esencias, ceras, resinas, flavonoides y carotenoides que brindan un efecto antioxidante.

Las principales propiedades medicinales atribuidas a la "pasuchaca" son: antidiabético, hipoglucemiante y depurador de la sangre; así mismo, se le considera un fuerte astringente, se suele emplear para combatir diarreas crónicas, cólera infantil, hemorragias, inflamaciones de la garganta y úlceras bucales. En la medicina homeopática se recomienda, además de los casos ya mencionados, en las blenorragias, diabetes, hemoptisis, menorragias, úlceras de las mamas y atonías del estómago y laringitis.

El efecto hipoglucemiante, según varios estudios realizados sobre esta planta, se ha podido verificar que la "pasuchaca" es la especie vegetal al que se atribuye como inhibidor de la $\alpha$-glucosidasa, la proteína que degrada el glucógeno en la síntesis de la glucosa. Entre sus propiedades antidiabéticas, estimula la secreción de la insulina por el páncreas con el fin de eliminar el nivel de azúcar en la sangre. Otro beneficio importante mediante el consumo de la "pasuchaca" es que inhibe la gluconeogénesis que es aumentada en los diabéticos o aumenta la captación de insulina por sus receptores. Por lo tanto teniendo en cuenta estas dos propiedades mencionadas anteriormente, esta planta está recomendada para el tratamiento de ciertos tipos de diabetes (diabetes tipo I y II). Además es un poderoso astringente, por lo que se utiliza para combatir la diarrea crónica, hemorragias, inflamaciones de la garganta, cólera infantil. Se utiliza desde hace muchos años por el ser humano sin que se produzcan efectos colaterales, se supone que su ingesta no implica riesgo para la salud. ${ }^{8}$

El objetivo de la presente investigación fue la detección, aislamiento y determinación estructural de los principales compuestos fenólicos del extracto hidroalcohólico de Geranium ruizii Hieron "pasuchaca"; se elaboró el extracto hidroalcohólico de la planta completa de "pasuchaca", se realizó su estudio fitoquímico y se aislaron compuestos fenólicos, se propone mediante espectroscopía ultravioleta visible la estructura de flavonoides.

\section{MATERIAL Y METODOS}

\section{Descripción botánica de la especie}

El Geranium ruizii Hieron "pasuchaca" es una planta inherente, acaule, silvestre que crece en forma espontánea. La raíz es pivotante, las hojas son basales pubescentes, sostenidas por peciolos (21 mm de largo), alternas, palmatipartidas en 7, dentibuladas. Los lóbulos ampliamente cuneados - abovados. Inflorescencia en umbela, pedúnculo, floral de $10 \mathrm{~mm}$ de largo. Su flor es periantica, diclamidea, heteroclamidea, hermafrodita, actinomorfa. Presenta en su base 5 verticilos de brácteas conolenas. Su fruto es un esquizocárpico de tipo regma que procede de un gineceo pluricarpelar con muchos estilos soldados entre sí, pero al madurar el fruto se separa cada uno con el correspondiente carpelo. ${ }^{6}$ (Figura $\left.N^{\circ} 1\right)$

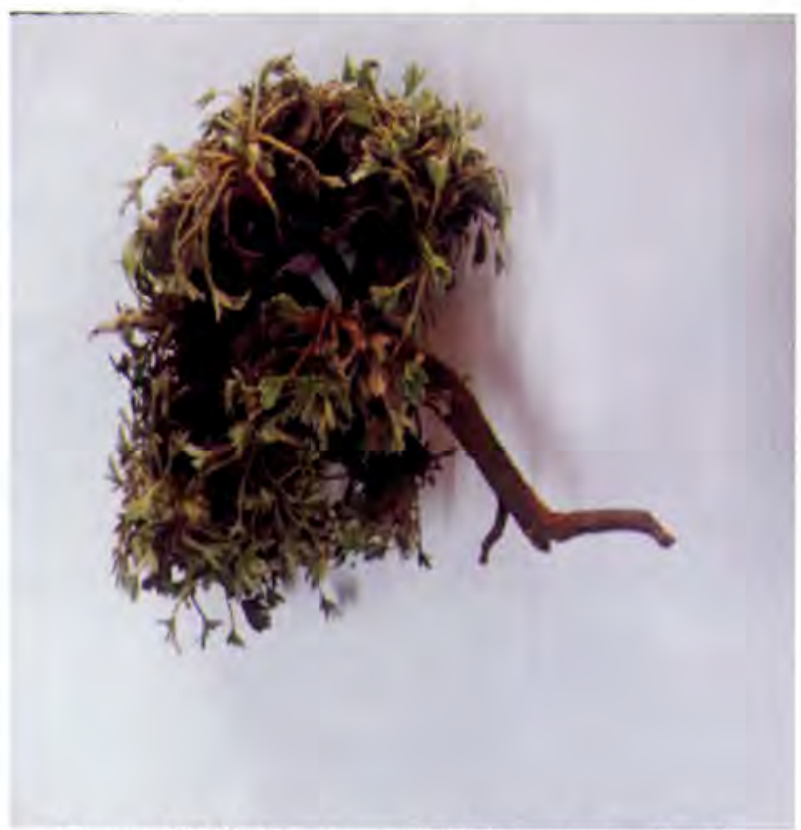

Figura N01. Geranium ruizii Hieron "pasuchaca".

\section{Preparación de la droga}

- Recolección de la planta completa, aproximadamente 5 kilos.

- Identificación taxonómica en el Museo de Historia Natural de la UNMSM, como Geranium ruizii Hieron "pasuchaca",

- Selección de muestras en buen estado, se utilizó toda la planta.

- Lavado y secado a temperatura ambiente de las plantas de Geranium ruizii Hieron "pasuchaca". 
Fernández G. et al., Determinación Estructural de Compuestos Fenólicos del Extracto Hidroalcohólico de Geranium ruizii Hieron

- Secado en estufa $\left(45^{\circ} \mathrm{C}\right)$ de las plantas de Geranium ruizii Hieron"pasuchaca".

- Reducción de tamaño del material vegetal en molino de cuchillas.

- Tamizado de la muestra (1 kilo en total) en mallas $\mathrm{N}^{\circ} 10$, $\mathrm{N}^{\circ} 30, \mathrm{~N}^{\circ} 40, \mathrm{~N}^{\circ} 50$ y $\mathrm{N}^{\circ} 80$ de Geranium ruizii Hieron "pasuchaca".

- Almacenamiento del polvo de Geranium ruizii Hieron "pasuchaca", en bolsas adecuadas. (Figura N²)

\section{Elaboración del extracto}

- Maceración hidroalcoholica al 50\%

- Los componentes solidos se colocan en un recipiente con el solvente y se deja reposar durante un periodo de 1 semana, agitándolo con frecuencia para mejor disolución de componentes solubles.

- Filtración al vacío asistido por una bomba de vacío.

- Secado hasta extracto seco en una estufa de aire circulante. Temperatura: $40^{\circ} \mathrm{C}$. (Figura $\mathrm{N}^{\circ}$ )

\section{Ensayo de solubilidad}

El extracto hidroalcohólico seco se trata con solventes de polaridad creciente. (Tabla $N^{\circ} 1$ )

\section{Tamizaje fitoquímico}

El tamizaje fitoquímico consiste en un conjunto de reacciones de coloración y precipitación que se realizan para detectar los componentes químicos del extracto hidroalcohólico de Geranium ruizii Hieron disuelto en agua destilada y en alcohol etílico $96^{\circ} .{ }^{10}\left(\mathrm{Tabla} \mathrm{N}^{\circ} 2\right)$

\section{Cromatografía en capa fina (CCF)}

Se realizó cromatografía en capa fina analítica del extracto hidroalcoholico, se sembró la muestra de extracto seco disuelto en agua destilada en el sistema de solventes de diclorometano: metanol (1:1). Se reveló la cromatoplaca de silicagel 60-G a la lámpara de luz UV $365 \mathrm{~nm}, 254 \mathrm{~nm}$ y $\mathrm{FeCl}_{3} \cdot{ }^{11}$ (Figura $\mathrm{N}^{\circ} 5$ y 6 )

\section{Cromatografía en capa fina a escala preparativa}

Posteriormente se realizó la cromatografía en capa fina a escala preparativa del extracto para aislar los componentes en el sistema de solventes de diclorometano: metanol (1:1). Se reveló la cromatoplaca de silicagel 60-G a la lámpara de luz UV $365 \mathrm{~nm}$ y $254 \mathrm{~nm}$. ${ }^{11}\left(\right.$ Figura N $^{\circ} 7$ )

Determinación de estructuras químicas

Luego de realizar la CCF a escala preparativa se

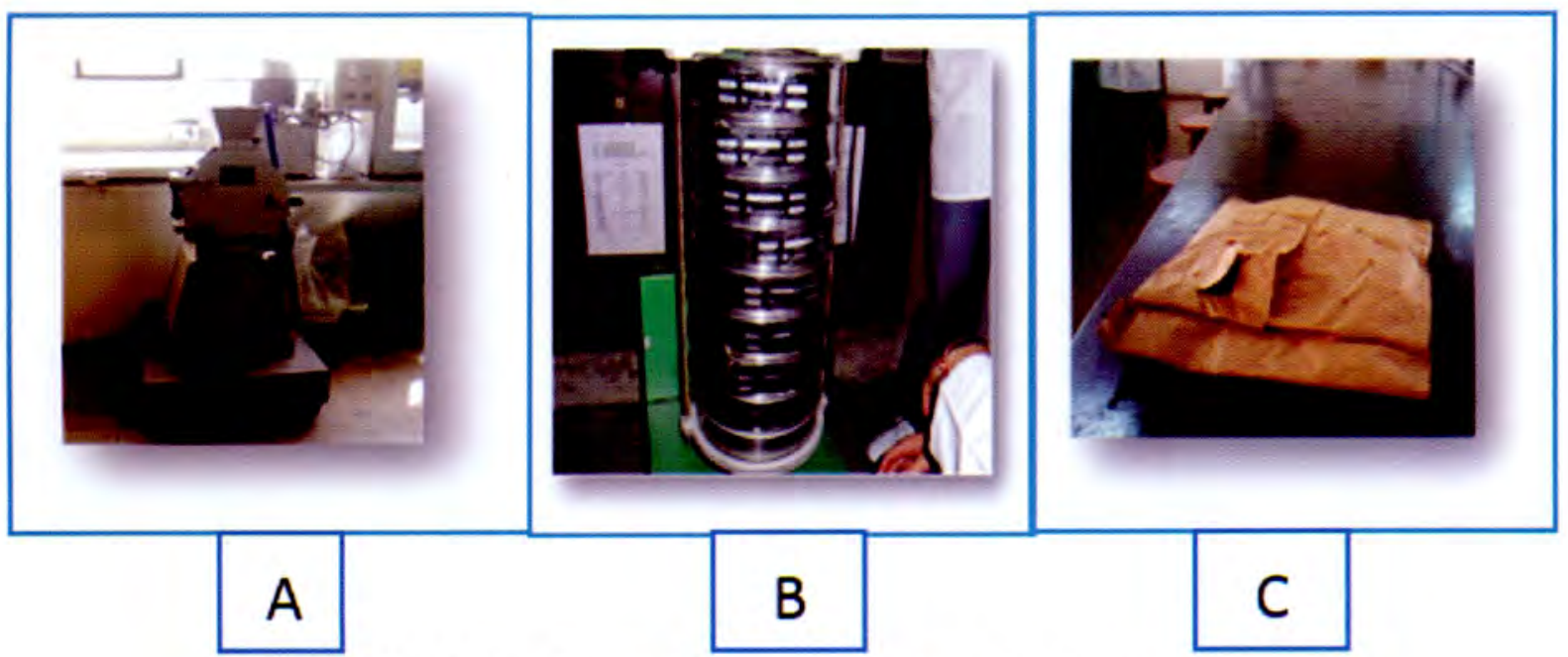

Figura $\mathrm{N}^{\circ}$ 2. A) Reducción de tamaño, B) Tamizado, C) Almacenamiento.
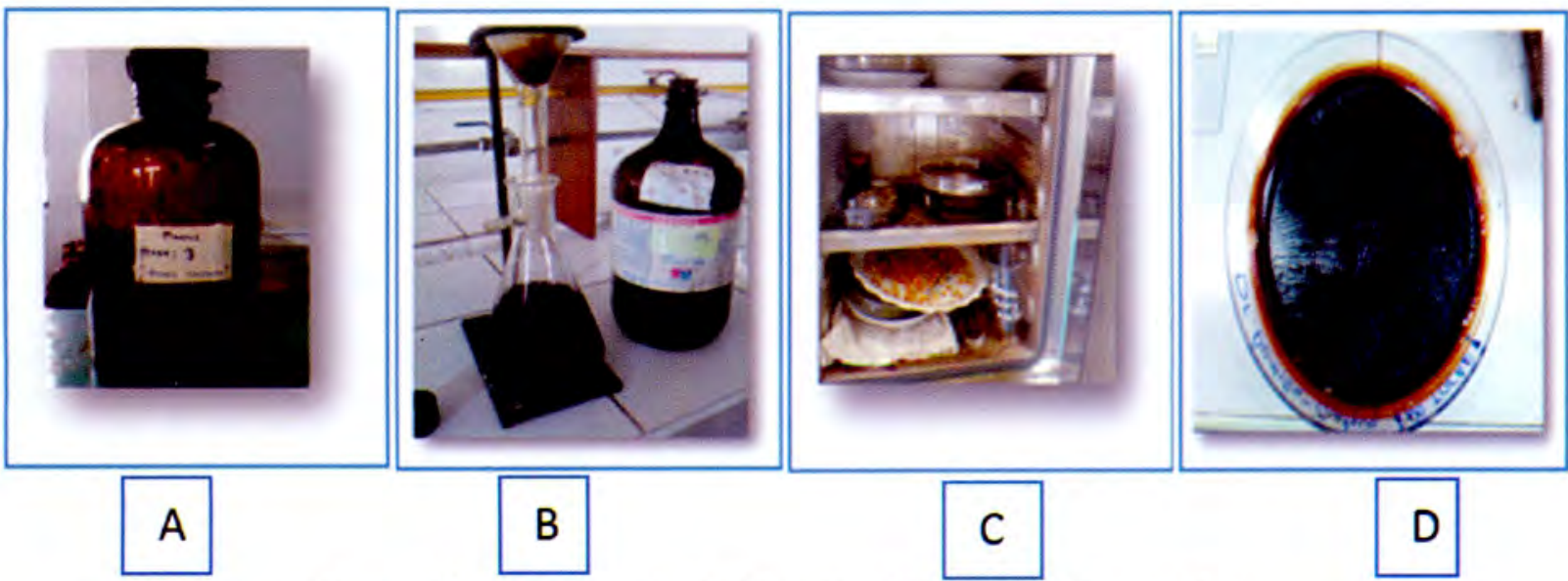

Figura N³. A) Maceración, B) Filtración, C) Secado, D) Extracto Seco. 
Fernández G. et al., Determinación Estructural de Compuestos Fenólicos del Extracto Hidroalcohólico de Geranium ruizii Hieron

procedió a la desorción de las manchas correspondientes. La silicagel con la mancha correspondiente se disolvió en metanol Q.P. y se centrifugó. Para separar los componentes se usó la centrífuga modelo Rotomix 32 a 2000 RPM durante 5 minutos, tomándose el sobrenadante y vertiéndolo en viales debidamente rotulados, los que fueron leídos en el espectrofotómetro UV/ vis $^{9,10}$.

\section{RESULTADOS Y DISCUSIÓN}

El Geranium ruizii Hieron "pasuchaca" es una planta silvestre, se recolectó $5 \mathrm{~kg}$ de la planta completa, identificada taxonómicamente en el Museo de Historia Natural de la UNMSM, luego de la selección de muestras de la planta completa en buen estado, se procedió al lavado y secado en estufa de aire circulante a $45^{\circ} \mathrm{C}$, se redujo de tamaño del material vegetal en molino de cuchillas, obteniéndose $1 \mathrm{~kg}$ del polvo que fue tamizado en mallas $\mathrm{N}^{\circ} 10, \mathrm{~N}^{\circ} 30, \mathrm{~N}^{\circ} 40, \mathrm{~N}^{\circ} 50$ y $\mathrm{N}^{\circ} 80$. Se realizaron 10 tamizados y se obtuvo como peso total 874,19 gramos de polvo tamizado, se procedió a su almacenamiento para su estudio posterior.

Elaboración del extracto, se realizó una maceración hidroalcohólica al $50 \%$, con los $874,19 \mathrm{~g}$ del polvo, durante 7 días con agitación periódica, luego se filtró al vacío para posteriormente concentrarlo a sequedad en una estufa de aire circulante a la temperatura de $40^{\circ} \mathrm{C}$ obteniéndose un extracto hidroalcohólico seco de 46.47 gramos.

\section{Ensayo de solubilidad}

Tabla $\mathbf{N}^{\circ}$ 1. Resultados del ensayo de solubilidad de Geranium ruizii Hieron

\begin{tabular}{cc}
\hline SOLVENTE & RESULTADO \\
\hline 1.Agua & +++ \\
2.Metanol & ++ \\
3.Etanol & +++ \\
4.n-Butanol & - \\
5.Acetona & - \\
6.Acetato de etilo & - \\
7.Diclorometano & - \\
8. Cloroformo & - \\
\hline
\end{tabular}

Como se observa en la tabla $\mathrm{N}^{\circ} 1$, el extracto hidroalcohólico seco al tratarse con solventes de polaridad creciente, fue bastante soluble en los solventes polares e insolubles en solventes de mediana polaridad lo que indica que los componentes del extracto seco de Geranium ruizii Hieron, son de estructura química polar.

\section{Tamizaje fitoquímico}

El tamizaje fitoquímico realizado para detectar los componentes químicos presentes en el extracto hidroalcohólico de Geranium ruizii Hieron disuelto en agua destilada y en alcohol etílico $96^{\circ}$ (Tabla N ${ }^{\circ} 2$ ), mostró buena cantidad de glicósidos ó carbohidratos, compuestos fenólicos, flavonoides y alcaloides.

Para la identificación de carbohidratos dio mejores resultados al solubilizar el extracto en agua que en alcohol,
Tabla $\mathbf{N}^{\circ}$ 2. Resultados del tamizaje fitoquímico de Geranium ruizzii Hieron.

\begin{tabular}{|c|c|c|c|}
\hline \multirow{2}{*}{ Metabolito } & \multirow{2}{*}{ Reacción } & \multicolumn{2}{|c|}{ Cantidad } \\
\hline & & $\mathrm{H}_{2} \mathrm{O}$ & $\mathrm{OH}$ \\
\hline \multirow[t]{3}{*}{ Carbohidratos } & Molish & ++ & ++ \\
\hline & Benedict & +++ & +++ \\
\hline & Fehling & +++ & ++ \\
\hline Comp. fenólicos & $\mathrm{FeCl}_{3}$ & +++ & + \\
\hline \multirow[t]{2}{*}{ Taninos } & Gelatina & +++ & + \\
\hline & Agua de Bromo & +++ & - \\
\hline $\begin{array}{l}\text { Triterpenoides y } \\
\text { esteroides }\end{array}$ & Lieberman-Burchard & ++ & ++ \\
\hline Flavonoides & Shinoda & +++ & ++ \\
\hline glicósidas & Baljet & ++ & - \\
\hline $\begin{array}{l}\text { Naftaquinonas, } \\
\text { antraquinonas y } \\
\text { antronas }\end{array}$ & Borntrager & +++ & +++ \\
\hline
\end{tabular}

Aminoácidos libres y Ninhidrina

grupos amino

\begin{tabular}{llcc} 
Alcaloides & Dragendorff & ++ & - \\
& Mayer & ++ & - \\
& Bertrand & ++ & - \\
& Sonnenschein & +++ & - \\
& Popoff & - & - \\
Saponinas & Saponinas & ++ & - \\
\hline
\end{tabular}

+++ :Abundante; ++: bastante; +: regular; -: ausencia

debido a que son compuestos polares al igual que los compuestos fenólicos, taninos, flavonoides, alcaloides y saponinas. Para antraquinonas dio los mismos resultados es decir los compuestos se solubilizan por igual y con la reacción de ninhidrina salió negativo debido a la ausencia de aminoácidos libres en el extracto.

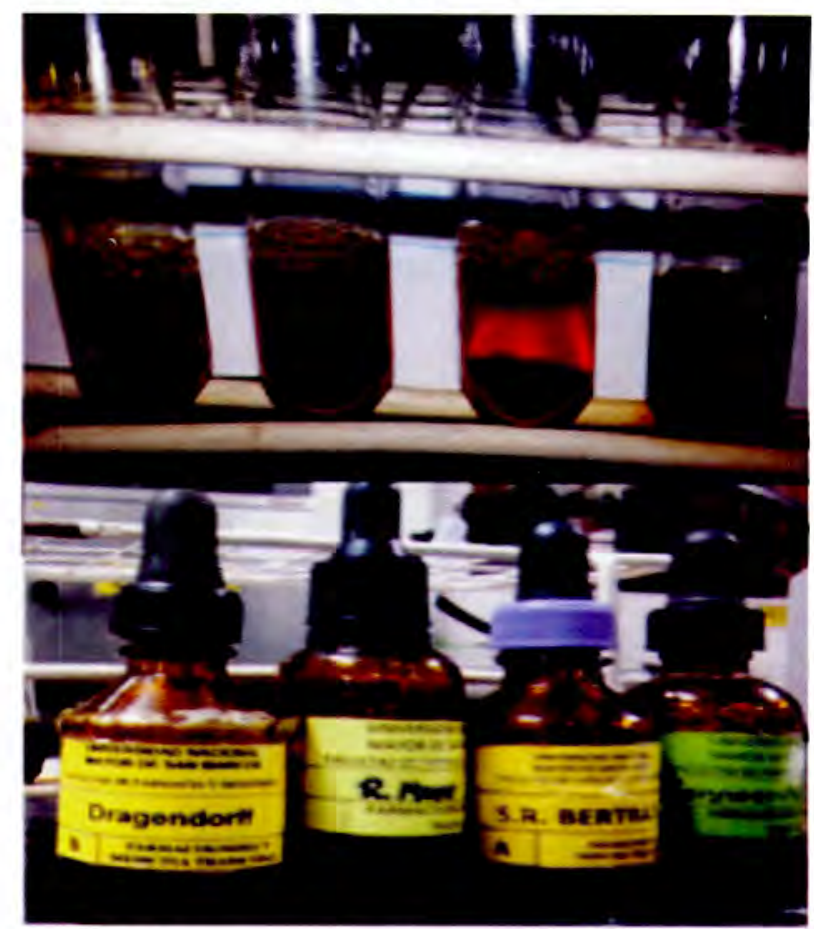

Figura $\mathrm{N}^{\circ} 4$. Reconocimiento de alcaloides 


\section{Cromatografia en capa fina}

\section{Revelado con luz Uv}

\begin{abstract}
Diclorometano: metanol (1:1) en la luz UV $365 \mathrm{~nm}$ y $254 \mathrm{~nm}$.
\end{abstract}

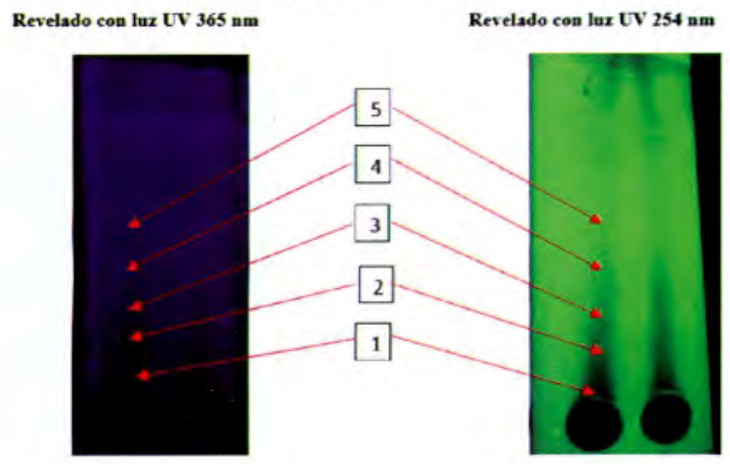

Figura $\mathbf{N}^{\circ}$ 5. Cromatografía en capa fina analítica del extracto hidroalcohólico de Geranium ruizii Hieronen revelado a la luz UV $365 \mathrm{~nm}$ y $254 \mathrm{~nm}$.

Como se ve en la Figura $\mathrm{N}^{\circ} 5$ con la cromatografía en capa fina analítica se visualizó la cantidad de componentes presentes en el extracto con el sistema de solventes diclorometano: metanol (1:1), al revelarse a la luz UV 365 y $254 \mathrm{~nm}$, observándose 5 manchas correspondientes a 5 componentes, las que luego de ser revelados con el reactivo de tricloruro de fierro, Figura $\mathrm{N}^{\circ} 6$, se observan tres manchas de color azul violáceo lo que nos indica la presencia de tres compuestos fenólicos.

\section{Revelado con tricloruro férrico $\left(\mathrm{FeCl}_{3}\right)$}

Diclorometano: metanol (1:1) revelado con $\mathrm{FeCl}_{3}$

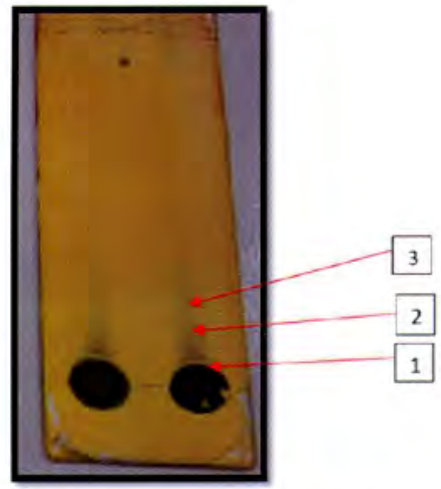

Figura $\mathbf{N}^{\circ}$ 6. Cromatografía en capa fina analítica del extracto hidroalcohólico de Geranium ruiz̨ii Hieron con revelador $\mathrm{FeCl}_{3}$.

\section{Cromatografia preparativa}

Después de detectar los 5 componentes mediante cromatografía en capa fina analítica, se procedió a aislar cada uno de ellos mediante cromatografía en capa fina preparativa en el sistema de solventes diclorometano: metanol(1:1) Figura $\mathrm{N}^{\circ} 7$, para su posterior desorción y lectura en el espectrofotómetro UV.
Diclorometano: metanol (1:1) en la luz UV $365 \mathrm{~nm}$ y $254 \mathrm{~nm}$.

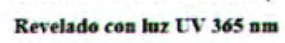

Revelado con luz UV $254 \mathrm{~nm}$
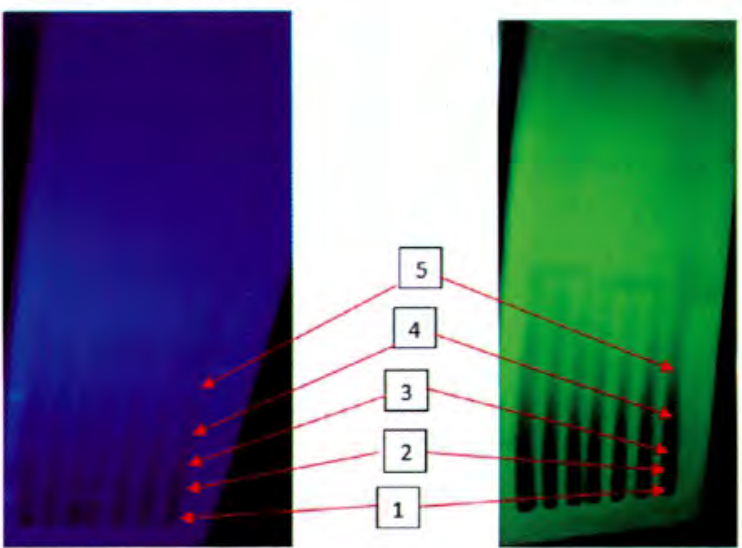

Figura $\mathbf{N}^{\circ}$ 7. Cromatografia en capa fina preparativa del extracto hidroalcohólico de Geranium ruizii Hieron, revelado a la luz UV $365 \mathrm{~nm}$ y $254 \mathrm{~nm}$.

\section{Propuesta de estructuras químicas}

Se realizó la desorción de los componentes aislados del extracto hidroalcohólico de pasuchaca, fueron analizados mediante espectroscopía UV, en un rango de 240-360 $\mathrm{nm}$ usando el equipo Thermoscientific modelo Helios Zeta y el programa Vision lite ${ }^{9.10}$, en el Centro de Producción Farmacéutica (CENPROFARMA) de la Facultad de Farmacia y Bioquímica de la UNMSM, y luego comparados con los resultados publicados por Mabry T. et al (1970) proponiéndose las siguientes estructuras: Figuras 810.<smiles>COc1cc(O)c2c(c1O)CC(c1ccccc1)=CC2=O</smiles>

Figura $\mathrm{N}^{\circ} 8 . \mathrm{N}^{\circ} 1 \quad \lambda_{\max }^{\mathrm{MeOH}} 251,276,308 \mathrm{~nm}$<smiles>COc1cc(O)c2c(c1OC)CC(c1ccccc1)=CC2=O</smiles>

Figura $\mathrm{N}^{\circ}$ 9. $\mathrm{N}^{\circ}$ 2. $\lambda_{\max }^{\mathrm{MeOH}} 274,308 \mathrm{~nm}$<smiles>O=C1C=C(c2ccc(O)cc2)Cc2c(O)c(O)cc(O)c21</smiles>

Figura $\mathrm{N}^{\circ} 10 . \mathrm{N}^{\circ}$ 3. $\lambda_{\text {máx }}^{\mathrm{MeOH}} 280,325 \mathrm{~nm}$ 
Fernández G. et al., Determinación Estructural de Compuestos Fenólicos del Extracto Hidroalcohólico de Geranium ruizii Hieron

\section{CONCLUSIONES}

1. Se determinó la presencia en el extracto hidroalcohólico de Geranium ruizii Hieronen de constituyentes químicos importantes como compuestos fenólicos, flavonoides, alcaloides y saponinas.

2. Mediante cromatografia en capa fina analítica y preparativa, en el sistema de solventes diclorometano:metanol (1:1) se obtuvo cinco componentes que se detectaron a la luz UV $254 \mathrm{~nm}$ y $365 \mathrm{~nm}$.

3. Mediante cromatografia en capa fina usando el revelador tricloruro férrico $\left(\mathrm{FeCl}_{3}\right)$ se detectaron tres compuestos fenólicos las que fueron confirmados mediante espectroscopía Ultravioleta, proponiéndose como flavonoides.

\section{REFERENCIAS BIBLIOGRÁFICAS}

1. Stewart GL, Tambascia M, Guzmán JR, Etchegoven F, Carrión JO, Artemenko S. Control of type 2 diabetes in private practice in nine countries of Latin America. Rev Panam Salud Publica. 2007; 22(1):12-20.

2. Organización Mundial de la Salud.Temas de Salud. Diabetes. OMS 2014. Disponible en:

http://www.who.int/topics/diabetes_mellitus/es/. Consultado en Abril 19 del 2014.

3. Huaracha Bedregal M J, Camiña Quispe L. Toxicidad aguda y efecto hipoglicemiante de Geranium dielianum Knuth 1991.

4. Cuerda C, Luengo L M, Valero M A, Vidal A, Burgos R, Calvo F L et al. Antioxidantes y diabetes mellitus: revisión de la evidencia. Nutr. Hosp. [revista en la
Internet]. 2011 Feb [citado 2014 Abr 20]; 26(1): 68-78. Disponible en:

http://scielo.isciii.es/scielo.php?script=sci_arttext\&pi $\mathrm{d}=$ S0212-16112011000100007\&lng=es

5. Conesa González A I, González Calero T M. Aspectos más recientes en relación con la diabetes mellitus tipo MODY. Rev Cubana Endocrinol [revista en la Internet]. 2012 Ago [citado 2014 Abr 19]; 23(2): 186-194. Disponible en:

http://scielo.sld.cu/scielo.php?script=sci_arttext\&pid $=$ S1561-29532012000200008\&lng $=$ es

6. Peralta G, Amaro E. Comercialización de plantas medicinales del Cuzco (Tesis de título), Lima - Perú: Universidad Nacional Mayor de San Marcos. 1990. p102.

7. Acosta M. Vademecum de plantas medicinales del Ecuador. $2^{a}$ ed. Quito: Abya-yala. Fundación de estudios sociales en Quito. 1999.p 37-42.

8. Camiña M, Huaracha L. Toxicidad aguda y efecto hipoglucemiante de Geranium dielsianum Knuth "pasuchaca" (Tesis de Título), Lima - Perú: Universidad Nacional Mayor de San Marcos. 1991. P120.

9. Mabry TJ, Markham KR, Thomas MB. The systematic identification of flavonoids. Springer-Verlag. New York-Heidelberg-Berlin, 1970.

10. Lock O. Investigación fitoquímica. Métodos en el estudio de productos naturales. $2^{a}$ ed. Lima. Pontificia Universidad Católica del Perú Fondo Editorial. Lima; 1994.p. 98-102.

11. Stahl E. Thin-Layer Chromatography. A Laboratory Handbook. Berlin. Editorial Springer-Velarg; 1978. p 687-703.

\begin{tabular}{ll}
\hline Correspondencia: & \\
Gustavo Adolfo Fernández Rebaza: & Fecha de Recepción: 06/10/2014 \\
gustav.unmsm@gmail.com & Fecha de Aceptación: 03/12/2014 \\
Jr. Puno 1002-Limal; Lima &
\end{tabular}

